\title{
THE CHICAGO CONFERENCE AND SEMINAR ON ALGEBRA
}

This seminar and conference were the principal features of the 1938 Summer Quarter program of the Department of Mathematics, University of Chicago, a program intended to emphasize algebra. The seminar began during the week preceding the conference with the following series of foundation lectures: The structure of algebras by A. A. Albert; Lie algebras by Nathan Jacobson; Valuations and lattices by Saunders MacLane; Introduction to the arithmetics of algebras by A. A. Albert; Arithmetic and class-field theory by Ralph Hull; Formal power series and polynomial ideals by Saunders MacLane.

The conference opened at 9:30 A.M., Tuesday, June 27, 1938. H. G. Gale, Dean of the Division of Physical Sciences of the University of Chicago, welcomed the group of 110 in attendance. A. A. Albert spoke on the purposes of the conference. The first lecture was given by L. E. Dickson (Chicago) who traced the history of the integral quantities of algebras. He described the difficulty of obtaining a non-vacuous definition of integral sets of algebras over the rational field and gave his final successful definition. He concluded with a statement of the structure theorems on algebras and the parallel results on their arithmetics. Ralph Hull (Illinois) followed with a description of his recent determination of explicit integral sets of cyclic algebras of prime degree. He also discussed recent work completely determining the class number of normal division algebras of degree greater than two over algebraic number fields, the consequent types of integral sets existing in such algebras, and the theory of their units.

In the afternoon session on Tuesday, A. A. Albert (Chicago) described some of the recent developments in the determination of the structure of normal division algebras, stressing the importance of cyclic algebras. He gave an outline of his recent new proofs of the fundamental properties of cyclic algebras. Richard Brauer (Toronto) then discussed his theory of the factor sets of arbitrary normal division algebras. He showed how these results provide a new method for studying the structure of these algebras, with particular success in the case of algebras of degree five.

The first Wednesday morning lecture was that of Saunders MacLane (Chicago). He formulated the elements of the theory of valuations of fields and discussed his results in connection with the question as to how the structure of a field complete with respect to a discrete valuation is determined by its residue-class field. He also connected the theory of valuations with the two following algebraic-geometric lectures. The first of these, by Oscar Zariski (Johns Hopkins), was on his solution by the use of ideals and valuation theory of the problem of the reduction of the singular points of algebraic surfaces. The second, by Solomon Lefschetz (Princeton), was the only lecture of the afternoon session. He indicated how many of the theorems on algebraic curves, that is on algebraic functions of one variable, which are usually obtained analytically can be obtained more easily by the use of purely algebraic devices. Moreover, the results are then valid for algebraic function fields whose constant coefficient field is other than the complex number field. The discussion of these two papers on applications of algebra was extremely lively.

The Department of Mathematics gave its Summer Quarter dinner Wednesday evening. It was attended by students and faculty as well as those attending the conference. There were 144 present, a group larger than at any previous mathematical dinner at Chicago. 
C. C. MacDuffee (Wisconsin) began the Thursday morning session with a discussion of the regular representations of an algebra by matrices. He discussed the correspondence of ideals in a maximal order with certain matrices, and indicated a matrix treatment of the question of the equivalence of ideals. C. G. Latimer (Kentucky) discussed the extensive arithmetic theory of quaternion algebras. He particularly emphasized the connections of ideals with classes of Hermitian forms.

Emil Artin (Notre Dame) opened the afternoon session on Thursday with a discourse on quadratic forms in $n$ variables. He connected the theory with that of the quadratic splitting fields of quaternion algebras, and sketched a new proof of Hasse's $p$-adic criterion that such a form be a null form. He also gave some results on quadratic forms over a field of characteristic two. Reinhold Baer (North Carolina) followed, speaking on the question as to how much of a group is determined by a given lattice of subgroups. His most complete results were for the case of abelian groups.

The first speaker on Friday morning was John Williamson (Johns Hopkins). He discussed the reduction of normal matrices to canonical form, and showed how the specializations of the type of normality lead to many classical reductions. M. H. Ingraham (Wisconsin) then discussed the matrix equation $B T=T A$ for matrices with elements in a division algebra. This is a generalization of the theory of the similarity of such matrices.

The final session, on Friday afternoon, began with the lecture of O. F. G. Schilling (Johns Hopkins). He showed how many of the theorems on $p$-adic number fields and their algebraic extensions hold for fields complete with respect to more general valuations. He also applied the theory to a study of normal division algebras over such fields. Nathan Jacobson (North Carolina) then discussed the theory of Lie algebras, in particular simple Lie algebras, over any non-modular field. He told how such algebras may be determined as certain subsets of associative algebras, in particular, of division algebras with an involutorial anti-automorphism.

In the seminar after the conference much of the conference material was discussed in detail in lectures by A. A. Albert and O. F. G. Schilling. In addition Sam Perlis (Chicago) gave his results on the integral sets of rational normal division algebras of composite degree, and Leonard Tornheim (Chicago) gave his work on the integral sets of quaternion algebras over function fields. Saunders MacLane spoke on $p$-bases of fields of characteristic $p$ and his results on the separability of such fields. D. M. Dribin (National Research Fellow) discussed the class-field theory for nonnormal fields. Nathan Jacobson gave the material of his paper on locally compact totally disconnected fields. There was also a long series of lectures by O. F. G. Schilling on generalized algebraic functions.

A list of references and a more complete discussion of some of the material of the conference will be found in Saunders MacLane's paper entitled Some recent advances in algebra, which is to be published in the American Mathematical Monthly.

A. A. Albert 